\title{
Pierre Simon Laplace 1749-1827. A Determined Scientist
}

Isabelle Laboulais

\section{(2) OpenEdition \\ 12 Journals}

\section{Édition électronique}

URL : https://journals.openedition.org/ahrf/8903

DOI : 10.4000/ahrf.8903

ISSN : 1952-403X

Éditeur :

Armand Colin, Société des études robespierristes

\section{Édition imprimée}

Date de publication : 1 mars 2007

Pagination : 218-220

ISSN : 0003-4436

\section{Référence électronique}

Isabelle Laboulais, «Pierre Simon Laplace 1749-1827. A Determined Scientist », Annales historiques de la Révolution française [En ligne], 347 | janvier-mars 2007, mis en ligne le 21 juillet 2008, consulté le 23 avril 2022. URL : http://journals.openedition.org/ahrf/8903 ; DOI : https://doi.org/10.4000/ahrf.8903

Ce document a été généré automatiquement le 23 avril 2022.

Tous droits réservés 


\title{
Pierre Simon Laplace 1749-1827. A Determined Scientist
}

\author{
Isabelle Laboulais
}

\section{RÉFÉRENCE}

Roger Hahn, Pierre Simon Laplace 1749-1827. A Determined Scientist, Cambridge (Mas.), Harvard University Press, 2005, 310 p., ISBN 0-674-01892-3, 32.30€.

1 S'il fallut attendre vingt-deux ans pour que le grand livre de Roger Hahn consacré à l'Académie des sciences de Paris soit traduit en français, force est de constater que sa biographie de Laplace a suivi un circuit de diffusion très différent (The Anatomy of a Scientific Institution. The Paris Academy of Science, 1666-1803, Berkeley, 1971; trad. fr. L'anatomie d'une institution scientifique: l'Académie des sciences de Paris, 1666-1803, ParisBruxelles, Éditions des Archives contemporaines, 1993). D'abord publié en français, en 2004, sous le titre Le système du monde. Pierre Simon Laplace, un itinéraire dans la science (Paris, Gallimard, Bibliothèque des histoires, 2004, 304 p.), ce texte a paru dès l'année suivante dans une version anglaise. Ces deux volumes diffèrent peu. Dans les deux cas, le texte a été établi par l'auteur et si ce dernier a pratiqué ici ou là quelques changements, il les qualifie lui-même de mineurs; le plan conçu en onze chapitres est resté absolument identique ; finalement, d'une version à l'autre, seule la structure des annexes varie. L'édition française offre en effet une bibliographie d'une vingtaine de pages que l'on ne retrouve pas dans l'édition anglaise. En revanche, celle-ci propose la transcription de plusieurs manuscrits utilisés par Roger Hahn (le brouillon d'une lettre que Jean-Étienne Guettard adresse à son jeune confrère au cours des années 1780, quatre manuscrits non scientifiques composés par Laplace et une note consacrée aux derniers moments de Laplace). Cette vingtaine de pages consacrée à la publication d'archives inédites reflète bien le point de vue radical de ce savant sur le christianisme - un point de vue quasiment absent de sa production imprimée et exprimé seulement en privé -, mais elle éclaire aussi (et surtout) la méthode mise en œuvre par l'historien des sciences américain pour revisiter la biographie de Laplace. Si les rares travaux 
jusqu'alors consacrés à ce savant s'appuient en effet sur ses œuvres imprimées, et notamment sur les quatorze volumes de ses œuvres complètes publiés entre 1878 et 1912 (c'est sur ce point notamment que l'approche de Roger Hahn se démarque de celle suivie par Charles Coulston Gillispie dans Pierre Simon Laplace 1749-1827. A Life in Exact Science, Princeton University Press, 1997, 322 p.), le livre de Roger Hahn repose, dans une large mesure, sur l'étude de la correspondance scientifique de Laplace patiemment réunie par l'historien et sur la consultation de documents privés restés longtemps inédits.

2 Ce corpus permet à Roger Hahn d'aborder la biographie de Laplace sous l'angle de l'histoire sociale des sciences, celle qu'il avait déjà mobilisée dans sa belle enquête consacrée à l'Académie des sciences. Cette fois, il revisite l'image historiographique de celui qui a souvent été regardé comme le Newton français. La biographie composée par Roger Hahn cherche à la fois à comprendre les enjeux et les circonstances qui ont guidé la carrière de Laplace et à présenter l'homme dans son contexte historique. Pour cela, l'historien de Berkeley propose une lecture chronologique de son parcours. Au fil des onze chapitres de son livre, il insiste non seulement sur son œuvre savante mais aussi sur l'ascension qui lui a permis de devenir un personnage clé de la politique scientifique conduite au tournant du XVIII ${ }^{e}$ et du XIX ${ }^{e}$ siècles.

3 Jusqu'en 1789, Laplace apparaît comme un homme prudent et mesuré, voire terne, un homme qui garde ses distances vis-à-vis de ses contemporains. Sa seule marque d'audace est cependant décisive puisqu'elle lui permet de sortir de son anonymat de jeune savant de province. À vingt ans, il écrit en effet à d'Alembert pour lui exposer ses travaux et cette rencontre lui ouvre les portes de l'Académie. C'est au sein de cette institution où il est élu à l'âge de vingt-quatre ans, qu'il établit un programme de travail englobant la mécanique céleste et la théorie des probabilités. Or, tout au long de sa carrière, il reste fidèle à ce cadre : il demeure convaincu que l'univers physique est régi par des principes invariables et cherche à établir la loi de la gravitation universelle grâce à des preuves empiriques. L'Académie des sciences lui offre non seulement d'exceptionnelles conditions de travail, mais elle lui permet de mettre en œuvre de fructueuses collaborations, notamment avec Lavoisier.

4 Avec la Révolution, le comportement de cet «homme tranquille» se modifie pourtant de manière notable, il devient peu à peu un personnage public et en vient même à symboliser la maîtrise scientifique. Roger Hahn le qualifie de «technocrate » lorsqu'il évoque son rôle dans l'établissement du système métrique décimal ; mais Laplace n'est pas l'homme d'un seul projet, on le retrouve dans toutes les institutions savantes marquantes de la période, de l'Institut à la Société d'Arcueil, en passant par l'École normale de l'an III et le Bureau des longitudes. Ces instances de savoir et de pouvoir lui donnent même l'occasion de devenir brièvement le ministre de l'Intérieur de Bonaparte, avant de gagner le Sénat puis la Chambre des pairs. Pour autant, Laplace ne délaisse jamais ses recherches et semble très attentif à leur diffusion. Outre les multiples éditions de son Exposition du système du monde et de son Essai philosophique sur les probabilités, il publie également de nombreux articles. Ses travaux et ses enseignements lui permettent de transmettre aux jeunes savants son inébranlable conviction: donner un sens mathématique aux phénomènes récurrents observés dans la nature.

Que deux historiens des sciences qui font autorité sur la connaissance de la fin du XVIII et du début du XIX siècle - Roger Hahn et Charles Coulston Gillispie - aient, en 
quelques années, consacré un livre au parcours de Laplace n'est pas anodin. Il s'agit à la fois d'un indice révélateur de la position centrale occupée par Laplace dans le monde des sciences et d'un signe du rôle nouveau joué par la biographie. Nous sommes loin ici du ton anecdotique ou de la biographie prétexte. Au contraire, Roger Hahn présente son livre comme l'œuvre d'une vie, comme le résultat d'un cheminement long d'un demi-siècle dans l'histoire des sciences. À le lire, on mesure en effet combien l'écriture biographique, dès lors qu'elle est conçue comme l'examen de ce que Pierre Bourdieu désignait comme le jeu des positions et des dispositions, peut offrir une synthèse sur une période ou un problème historique, en l'occurrence les figures de savants, du temps des Lumières à celui de la Révolution. 